\title{
SOX18 knockdown suppresses the proliferation and metastasis, and induces the apoptosis of osteosarcoma cells
}

\author{
ZHONG WU* , JUNJIAN LIU* , JIANGUANG WANG and FAN ZHANG \\ Department of Orthopedics, Shanghai Tenth People's Hospital, Tongji University School of Medicine, \\ Shanghai 200072, P.R. China
}

Received January 5, 2015; Accepted October 14, 2015

DOI: $10.3892 / \mathrm{mmr} .2015 .4541$

\begin{abstract}
Sex determining region Y-box 18 (SOX18) has been found to be overexpressed in several types of tumor. However, the molecular mechanism underlying the biological function of SOX18 in osteosarcoma remains to be elucidated. The present study aimed to elucidate the roles of SOX18 in regulating the biological behavior of osteosarcoma cells. First, SOX18 mRNA expression was analyzed in osteosarcoma tissues using reverse transcription-quantitative polymerase chain reaction (RT-qPCR). The results demonstrated that the expression of SOX18 was elevated in osteosarcoma tissue, compared with normal bone tissue. In addition, the knockdown of SOX18 in U2OS or MG63 osteosarcoma cells inhibited cell proliferation and significantly increased the population of cells in the S-phase of the cell cycle, as measured by the CCK- 8 assay and flow cytometric analysis, respectively. Additionally, suppression of the expression of SOX18 in the osteosarcoma cells significantly induced cell apoptosis as evaluated by annexin V/propidium iodide staining and flow cytometric analysis. The downregulation of SOX18 was found to significantly inhibit cell adhesion and invasion. The mRNA and protein expression levels of transforming growth factor- $\beta$, platelet-derived growth factor (PDGF)-A, PDGF-B and RhoA were also reduced by SOX18 silencing, as assessed by RT-qPCR and western blot analysis, respectively. These results indicated that SOX18 may function as an oncogene, and may provide a novel and promising therapeutic strategy for osteosarcoma.
\end{abstract}

Correspondence to: Dr Fan Zhang, Department of Orthopedics, Shanghai Tenth People's Hospital, Tongji University School of Medicine, 301 Yanchang Road, Shanghai 200072, P.R. China

E-mail: zhangf811@163.com

*Contributed equally

Key words: sex determining region Y-box 18, osteosarcoma, proliferation, apoptosis, invasion, transforming growth factor- $\beta 1$, Ras homolog family member A

\section{Introduction}

Osteosarcoma is the most common type of primary bone tumor worldwide and exhibits a peak incidence in the second and third decades of life (1). Osteosarcoma can arise in any bone, however, it is most common in the metaphyses of long bones (1). Although survival rates have increased between 20 and $-75 \%$ due to the combination of radical surgery and neoadjuvant chemotherapy (2-4), for patients who present with metastatic disease or present with tumor recurrence, the survival rates remain $<30$ and $<20 \%$, respectively (5). This emphasizes the requirement for the development of novel therapeutic targets and approaches for the treatment of osteosarcoma.

Sex determining region Y-box 18 (SOX18) is a member of the sex-determining region of the $\mathrm{Y}$ chromosome-related high mobility group box (SOX) family of transcription factors. It selectively interacts with the common SOX target sequence $(\mathrm{A} / \mathrm{T}) \mathrm{ACAA}(\mathrm{A} / \mathrm{T}) \mathrm{G}$, and activates transcription via a transactivation domain adjacent to the high mobility group domain $(6,7)$. Previous studies $(8,9)$ have suggested that the expression level of SOX18 may affect tumor growth. It has been reported that the expression levels of SOX18 are increased in gastric cancer tissues, compared with normal gastric tissues (10). Furthermore, the expression of SOX18 is correlated with poor survival rates (10). The expression of SOX18 has also been correlated with poor clinical outcome in patients with non-small cell lung cancer (11), ovarian cancer (12) and invasive ductal breast carcinoma (13). However, the expression pattern and biological functions of SOX18 in osteosarcoma remain to be fully elucidated.

The present study aimed to investigate the role of SOX18 in osteosarcoma. Initially, the expression levels of SOX18 were analyzed using reverse transcription-quantitative polymerase chain reaction ( $\mathrm{RT}-\mathrm{qPCR}$ ) analysis in osteosarcoma tissue samples obtained from 25 patients. Subsequently, the biological function of SOX18 in osteosarcoma cell lines was investigated using RNA interference (RNAi). The present study also aimed to elucidate whether SOX18 is involved in cell proliferation, cell cycle progression, apoptosis, adhesion and invasion, and whether SOX18 is involved in these processes by regulating the expression of transforming growth factor- $\beta 1$ (TGF- $\beta 1$ ), platelet-derived growth factor-A (PDGF-A), PDGF-B and Ras homolog family member A (RhoA). 


\section{Materials and methods}

Patients and tissue samples. Between 2010 and 2012, 25 patients (16 men and 9 women) with conventional (occurring in the metaphyses of the long bones) osteosarcoma, who were admitted to the Department of Orthopedics, Shanghai Tenth People's Hospital (Tongji University, Shanghai, China), were enrolled in the present study. Complete clinical and pathological follow-up data were obtained for all patients. The patients ranged in age from 7 to 49 years with a median age of 18 years. Osteosarcoma tissues (0.1-0.2 g) were obtained from femur or tibia of these 25 patients and normal bone tissues were also collected as negative controls. These normal bone tissues were resected within at least $5 \mathrm{~cm}$ of the tumor margin when the patients underwent definitive surgery. Ethical approval for the present study was provided by the independent ethics committee of Shanghai Tenth People's Hospital, Tongji University (Shanghai, China). Informed and written consent was obtained from all patients or their advisers, according to the ethics committee guidelines.

Antibodies. The following primary antibodies were used in the present study: Mouse polyclonal SOX18 (Ab66145; 1:1,000), rabbit polyclonal TGF- $\beta 1$ (Ab92486; $1: 400)$ and rabbit polyclonal RhoA (Ab68826; 1:2,000) (Abcam, Cambridge, MA, USA), rabbit polyclonal PDGF-A (BA0408; 1:200) and rabbit polyclonal PDGF-B (BA0519-2; 1:200) (Wuhan Boster Biological Technology, Ltd. (Wuhan, China) and rabbit monoclonal glyceraldehyde 3-phosphate dehydrogenase (GAPDH; \#5174; 1:2,000), Cell Signaling Technology, Inc. (Danvers, MA, USA). Horseradish peroxidase-conjugated goat anti-mouse and goat anti-rabbit secondary antibodies were purchased from Beyotime Institute Biotechnology (Shanghai, China).

Cell culture. MG63, HOS, 143B, Saos2, U2OS and HEK293T cells were purchased from the American Type Culture Collection (Rockville, MD, USA). The MG63, HOS, Saos2, 143B and HEK293T cells were grown in Dulbecco's modified Eagle's medium (DMEM; Thermo Fisher Scientific, Inc., Waltham, MA, USA) with $10 \%$ fetal bovine serum (FBS; Thermo Fisher Scientific, Inc.) and 1\% penicillin/streptomycin (Thermo Fisher Scientific, Inc.). The U2OS cells were grown in RPMI-1640 medium with 10\% FBS (Thermo Fisher Scientific, Inc.) and $1 \%$ penicillin/streptomycin. All cell lines were maintained at $37^{\circ} \mathrm{C}$ in a $5 \% \mathrm{CO}_{2}$ atmosphere.

Vector construction and virus transduction. Three shRNAs targeting human SOX18 mRNA (SOX18-Ri-3, AGGAAG CCGAACGGCTGCGTT; SOX18-Ri-2, AGGCTGCCTTCT TCCCTCCTT; and SOX18-Ri-3, TACCACGTGGCACTG GCCATT; Generay Biotech Co., Ltd., Shanghai, China) were cloned into a lentiviral vector (PLKO.1; Addgene, Inc., Cambridge, MA, USA). A non-specific scramble shRNA sequence (TTCTCCGAACGTGTCACGTTT) was used as the negative control (NC). The constructs were then transfected into the HEK293T cells with lentiviral packaging vectors using Lipofectamine 2000 (Invitrogen; Thermo Fisher Scientific, Inc.), according to the manufacturer's protocol. The viruses were collected $48 \mathrm{~h}$ subsequent to transfection and used to transduce the U2OS cells and MG63 cells. After $48 \mathrm{~h}$, the cells were processed for RT-qPCR and western blotting.

$R T-q P C R$. Total RNA was extracted using TRIzol reagent (Invitrogen; Thermo Fisher Scientific, Inc.), according to the manufacturer's protocol. Total RNA $(1 \mu \mathrm{g})$ was reverse transcribed using a First Strand cDNA Synthesis kit (K1612; Thermo Fisher Scientific Inc.), according to the manufacturer's protocol. RT-qPCR was performed using a SYBR Green PCR kit (Thermo Fisher Scientific, Inc.) on an ABI 7300 Real-time PCR machine (Applied Biosystems; Thermo Fisher Scientific, Inc., Foster City, CA, USA) using the following cycling parameters: $95^{\circ} \mathrm{C}$ for $10 \mathrm{~min}$, followed by 40 cycles of $95^{\circ} \mathrm{C}$ for $15 \mathrm{sec}$ and $60^{\circ} \mathrm{C}$ for $45 \mathrm{sec}$. GAPDH served as an internal control. The gene expression was calculated using the $\Delta \Delta \mathrm{Ct}$ method (14). All data represent the average of three replicates. The primers used (Generay Biotech Co., Ltd.) were as follows: SOX18 (NM_018419.2), forward 5'-CGCGTGTAT GTTTGGTTC-3' and reverse 5'-ATGTAACCCTGGCAA CTC-3'; TGF- $\beta 1$ (NM_000660.4), forward 5'-GACTACTAC GCCAAGGAGGTC-3' and reverse 5'-GAGAGCAACACG GGTTCAG-3'; PDGF-A (NM_002607.5), forward 5'-CGT AGGGAGTGAGGATTCTTTG-3' and reverse 5'-AAATGA CCGTCCTGGTCTTG-3'; PDGF-B (NM_002608.2), forward 5'-CTCGATCCGCTCCTTTGATG-3' and reverse 5'-AGG AAGTTGGCGTTGGTG-3'; RhoA (NM_001664.2), forward 5'-GAGTGTTCAGCAAAGACCAAAG-3' and reverse 5'-TTG CAGCAAGGTTTCACAAG-3'; GAPDH (NM_001256799.1), forward 5'-CACCCACTCCTCCACCTTTG-3' and reverse 5'-CCACCACCCTGTTGCTGTAG-3'.

Western blotting. The treated and untreated MG63 and U2OS cells were harvested and washed twice with phosphate-buffered saline (PBS) and lysed in ice-cold radio immunoprecipitation assay buffer (JRDUN Biotechnology Co., Ltd., Shanghai, China) with freshly added $0.01 \%$ protease inhibitor cocktail (Sigma-Aldrich, St. Louis, MO, USA). The cells were then incubated on ice for $30 \mathrm{~min}$. The cell lysates were centrifuged at $16,000 \times \mathrm{g}$ for $10 \mathrm{~min}$ at $4^{\circ} \mathrm{C}$. Protein concentration was measured using the Bicinchoninic Acid Assay kit (Thermo Fisher Scientific, Inc.) and the supernatant (20-30 $\mu$ g protein) was run on a 15\% SDS-PAGE gel and transferred electrophoretically onto a nitrocellulose membrane (EMD Millipore, Billerica, MA, USA). Subsequent to blocking with 5\% skimmed milk, the membranes were incubated with the primary antibodies, followed by the corresponding horseradish peroxidase-conjugated secondary antibodies (Beyotime Institute of Biotechnology). The blots were then visualized using enhanced chemiluminescence (EMD Millipore).

Cell proliferation assay. Cell proliferation was measured using a Cell Counting Kit-8 (CCK-8; Dojindo Molecular Technologies, Inc., Kumamoto, Japan) according to manufacturer's protocol. In brief, the U2OS and MG63 cells $\left(\sim 1-5 \times 10^{3}\right)$ were seeded into 96 -well plates. At 0, 24, 48 and $72 \mathrm{~h}$, CCK-8 solution (10 $\mu 1$ in $100 \mu 1$ DMEM) was added into each well, followed by incubation at $37^{\circ} \mathrm{C}$ for $1 \mathrm{~h}$. The optical density values were measured at a wavelength of $450 \mathrm{~nm}$ using a microplate reader (Model 550; Bio-Rad Laboratories, Inc., 
Hercules, CA, USA). All experiments were performed in triplicate and repeated a minimum of three times.

Cell cycle distribution analysis. Propidium iodide (PI; Sigma-Aldrich) staining was performed to analyze the DNA content in the cells to determine cell cycle distribution. The cells were harvested $48 \mathrm{~h}$ following transduction, and were labeled with PI, as previously described (15). In brief, the cells were resuspended in PBS and fixed with $70 \%$ ethanol. Cells were washed twice with PBS and then suspended at a concentration of $1 \times 10^{6}$ cells $/ \mathrm{ml}$. Following treatment with ribonuclease (Sigma-Aldrich) for $15 \mathrm{~min}$ at $37^{\circ} \mathrm{C}$, PI $(0.05 \mathrm{mg} / \mathrm{ml})$ was added to the cells, followed by incubation at room temperature in the dark for $30 \mathrm{~min}$. DNA content was then analyzed using a FACScan instrument equipped with FACStation running CellQuest software, version 3.3 (BD Biosciences, San Jose, CA, USA).

Cell apoptosis assay. The percentages of the cells actively undergoing apoptosis were determined by double staining with annexin V-fluorescein isothiocyanate (FITC) and PI. The adherent and floating virally transduced or control cells were harvested after $48 \mathrm{~h}$, and double-labeled with annexin V-FITC and PI (BD Biosciences), according to the manufacturer's protocol. The cells were analyzed using a FACScan instrument equipped with FACStation running CellQuest software.

Cell adhesion assay. To determine cell adhesion, the assay was performed in 12-well plates. The plates were pre-coated with $1 \mathrm{ml}$ fibronectin $(5 \mu \mathrm{g} / \mathrm{ml})$ for $2 \mathrm{~h}$ at room temperature. The cells were transduced, as described above, $48 \mathrm{~h}$ prior to the assay. The cells were seeded into the coated plates at a density of $10^{5}$ cells/well and allowed to adhere at $37^{\circ} \mathrm{C}$ for $1 \mathrm{~h}$. Non-adherent cells were washed off with PBS and the cells were fixed in $4 \%$ paraformaldehyde (Beijing Solarbio Science \& Technology Co., Ltd., Beijing, China) and stained with $0.2 \%$ crystal violet (Beijing Solarbio Science \& Technology Co., Ltd.). The number of adherent cells was determined in five randomly selected fields under a microscope (Eclipse E600; Nikon Corporation, Tokyo, Japan), as previously described (16).

In vitro invasion assay. The upper well of a Transwell chamber (Corning Incorporated, NY, USA) was coated with Matrigel (BD Biosciences) at $37^{\circ} \mathrm{C}$ in a $5 \% \mathrm{CO}_{2}$ incubator for $1 \mathrm{~h}$. The virus-treated and untreated cells were serum starved for $24 \mathrm{~h}$, then $500 \mu \mathrm{l}$ cell suspension containing $10^{5}$ cells $/ \mathrm{ml}$ were placed in the upper compartment of the chamber. Culture medium supplemented with $10 \%$ FBS $(750 \mu 1)$ was added into the lower well of the chamber. The cells were allowed to invade through the Matrigel membrane for $48 \mathrm{~h}$, and non-invasive cells were removed from the upper membrane. The invasive cells on the underneath were washed with PBS, fixed in $4 \%$ paraformaldehyde and stained with $0.2 \%$ crystal violet. The invading cells were observed under a microscope. Cells were counted in the central field of the membranes in triplicate.

Statistical analysis. All data are presented as the mean \pm standard deviation. Statistical significance was determined using
Student's two-tailed t-test with SPSS software, version 13.0 (SPSS, Inc., Chicago, IL, USA). $\mathrm{P}<0.05$ was considered to indicate a statistically significant difference.

\section{Results}

SOX18 is overexpressed in osteosarcoma. The mRNA levels of SOX18 were measured in the osteosarcoma and adjacent normal tissues of 25 patients using RT-qPCR. As presented in Fig. 1A, SOX18 was overexpressed in $88 \%$ (22/25) of the osteosarcoma tissues assessed. Statistical analysis using Student's t-test indicated that SOX18 mRNA was significantly overexpressed in osteosarcoma tissues, compared with normal tissues $(\mathrm{P}<0.001)$.

Knockdown of SOX18 suppresses the proliferation of osteosarcoma cells. The expression levels of SOX18 in five osteosarcoma cell lines, Saos2, U2OS, HOS, MG63 and 143B, were assessed using RT-qPCR and western blotting. The results demonstrated that two of these cell lines, MG63 and U2OS, exhibited higher mRNA and protein expression levels of SOX18, compared with the remaining Saos2, HOS and 143B cell lines, which exhibited lower mRNA and protein expression levels of SOX18 (Fig. 1B).

To investigate the effect of SOX18 on osteosarcoma, SOX18 was knocked down in osteosarcoma cells using RNAi. U2OS and MG63 cells were selected for the RNAi experiment due to the fact that they expressed higher levels of SOX18. Three pairs of shRNA (SOX18-Ri-3, SOX18-Ri-2 and SOX18-Ri-3) targeting human SOX18, and a non-specific scramble shRNA (NC) were designed and cloned into a lentiviral plasmid. The recombinant lentivirus was then packaged into the HEK293T cells and used to transduce the U2OS and MG63 cells. The silencing effect of the shRNA was evaluated by western blotting and RT-qPCR (Fig. 1C and D). The results indicated that SOX18-Ri-3 was the most efficient shRNA, with a knockdown efficiency of $\sim 70 \%$. Therefore, SOX18-Ri-3 was selected for the following assays.

The effect of SOX18 RNAi on the proliferation of osteosarcoma was then assessed. Knockdown of SOX18 by transduction of the SOX18-shRNA virus into the U2OS or MG63 cells resulted in a reduced cell growth rate, compared with the corresponding control (Fig. 1E and F), whereas a similar growth rate was observed between the WT cells and the NC cells. These results indicated that SOX18 may promote the proliferation of osteosarcoma cells.

Silencing of SOX18 induces S-phase arrest and apoptosis in osteosarcoma cells. The potential effects of SOX18 knockdown on cell cycle progression were then investigated. PI staining and flow cytometry analysis revealed that knockdown of SOX18 in the U2OS (Fig. 2A) and MG63 cells (Fig. 2B) resulted in an increase in the number of cells in the S-phase and a corresponding reduction in the number of cells in the $\mathrm{G}_{0} / \mathrm{G}_{1}$-phase. These results suggested that silencing of SOX18 prevented the osteosarcoma cells from entering the $\mathrm{G}_{2} / \mathrm{M}$-phase.

The apoptotic function of SOX18 in U2OS and MG63 cells was also assessed using the annexin V-FITC/PI staining assay. As shown in Fig. 2C and D, flow cytometric analysis 
A

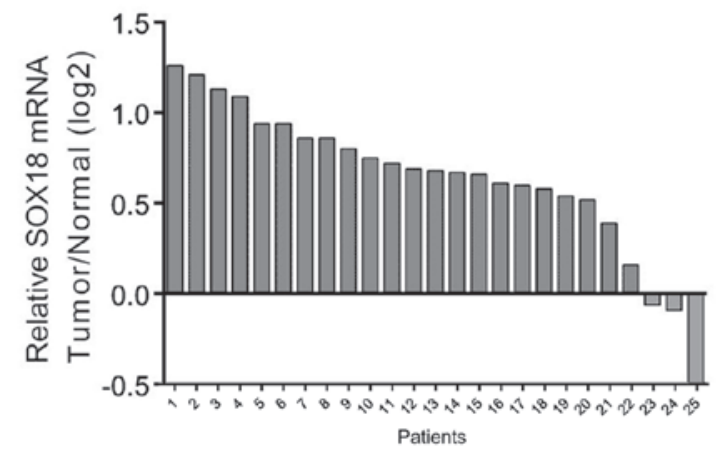

C

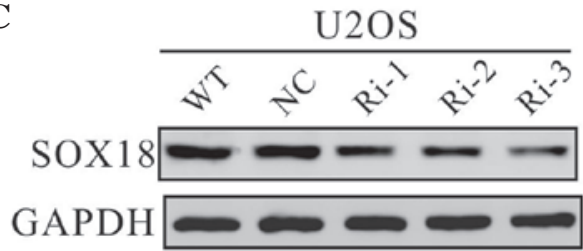

$\mathbf{E}$

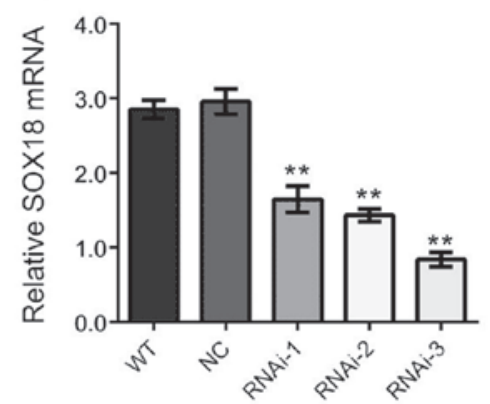

\section{U2OS}

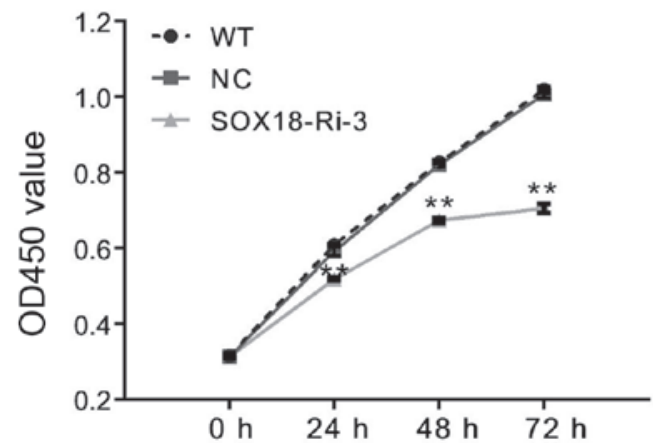

B

SOX18
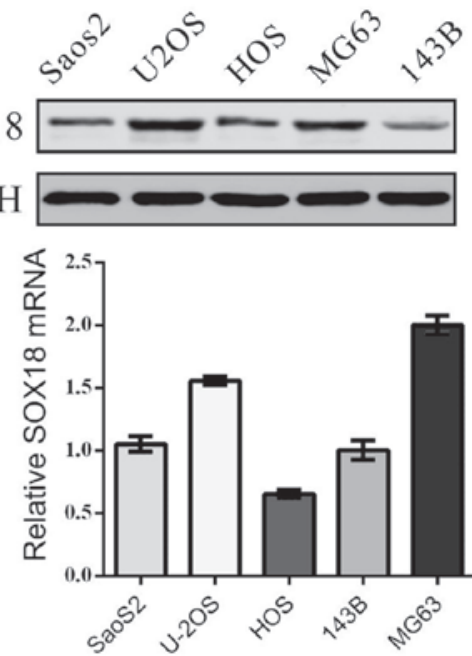

D

MG63

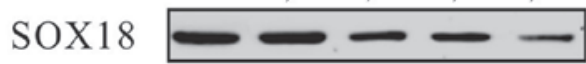

GAPDH

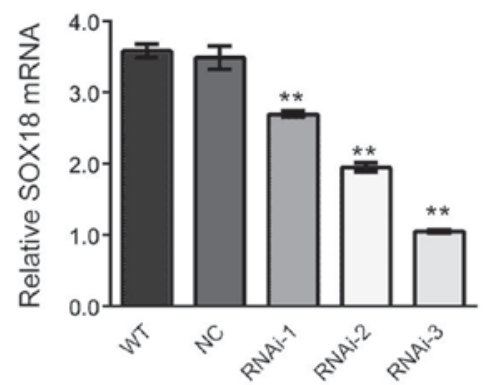

$\mathbf{F}$

\section{MG63}

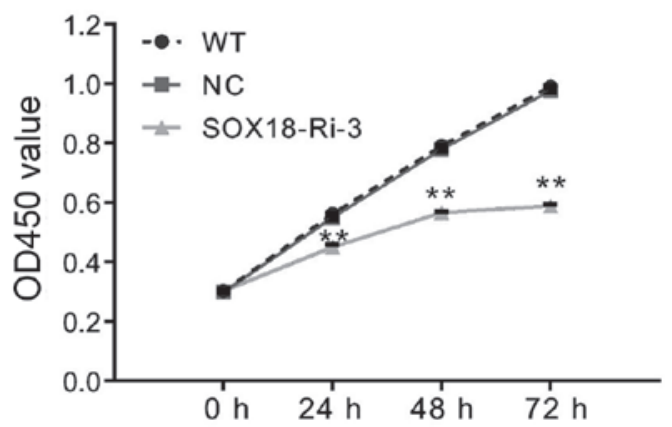

Figure 1. SOX18 is overexpressed in osteosarcoma tissues, and the knockdown of SOX18 suppresses the proliferation of osteosarcoma cells. (A) mRNA expression levels of SOX18 were significantly increased in the osteosarcoma tissues $(\mathrm{n}=25)$, compared with the levels in the normal tissues $(\mathrm{n}=25)$, obtained from patients admitted to the Shanghai Tenth People's Hospital (Shanghai, China) between 2009 and 2012. In the graph, a positive log2 Tumor/Normal ratio on the $y$-axis indicates increased expression levels of SOX18 in the tumor tissue, whereas a negative log2 Tumor/Normal ratio indicates reduced expression levels of SOX18 in the tumor tissue. (B) Expression levels of SOX18 in five osteosarcoma cell lines were analyzed using western blotting (upper panel) and RT-qPCR (lower panel). Data are representative of three independent experiments. (C and D) Expression levels of SOX18 in U2OS and MG63 cells was analyzed using western blotting (upper panel) and RT-qPCR (lower panel). (E and F) Cell proliferation was detected 24, 48 and $72 \mathrm{~h}$ subsequent to viral transduction of the U2OS and MG63 cells. Data are representative of three independent experiments and are presented as the mean \pm standard deviation ( ${ }^{* *} \mathrm{P}<0.01$, vs. NC). SOX18, sex-determining region Y-box 18; GAPDH, glyceraldehyde 3-phosphate dehydrogenase; WT, wild-type; NC, scrambled shRNA; RNAi-1, SOX18-shRNA-1 virus transduction; SOX18-Ri-3, SOX18-shRNA-3 virus transduction; OD, optical density; RT-qPCR, reverse transcription-quantitative polymerase chain reaction.

demonstrated that knockdown of SOX18 in the U2OS or MG63 cells significantly induced cell apoptosis, compared with the corresponding scramble shRNA (U2OS cells, $23.03 \pm 0.46$, vs.
$3.80 \pm 0.23 \%$; MG63 cells, $34.43 \pm 1.32$, vs. $3.93 \pm 0.18 \%$ ). These results indicated that the proliferation-promoting function of SOX18 may be mediated via the promotion of cell cycle 
A
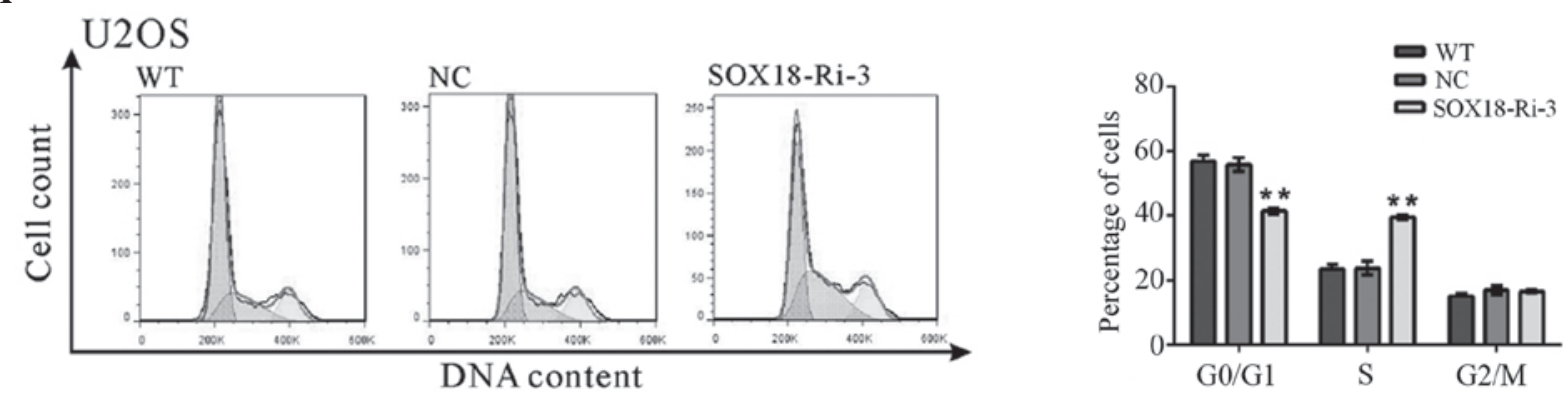

B
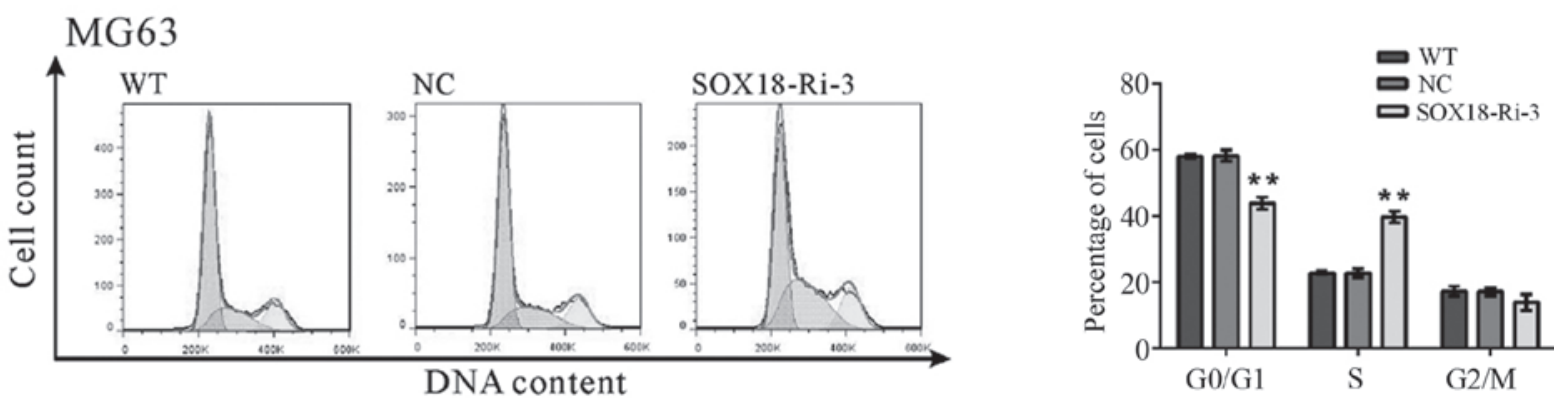

C

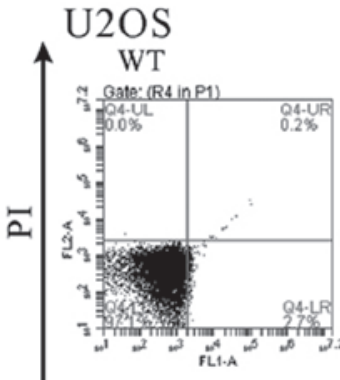

NC

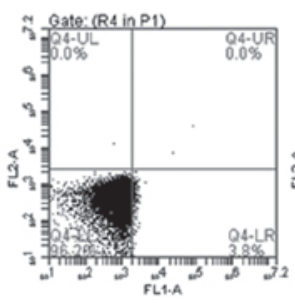

Annexin V-FITC

D

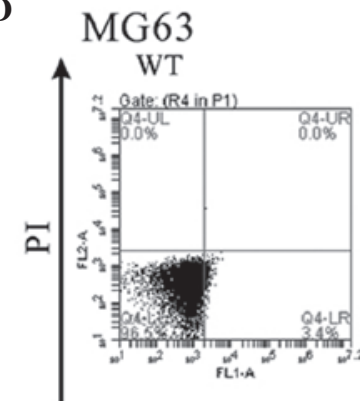

NC

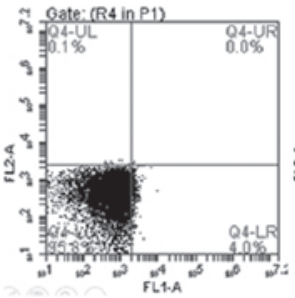

SOX18-Ri-3

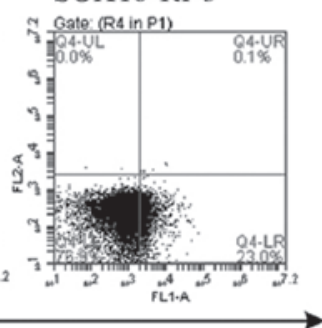

SOX18-Ri-3

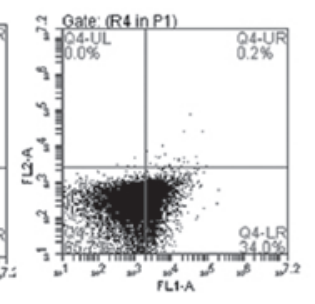

Annexin V-FITC
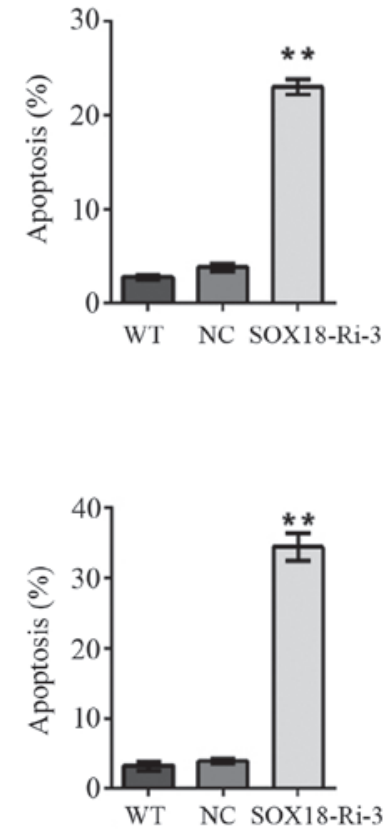

Figure 2. SOX18 RNAi induces S-phase arrest and apoptosis in osteosarcoma cells. The U2OS and MG63 cells were transduced with the indicated virus and were collected after 48 h. (A and B) Cell cycle profile was analyzed using flow cytometry. (C and D) Cells were stained with annexin V-FITC/PI, and apoptotic rates was analyzed using flow cytometry. Data are representative of a minimum of three independent experiments and are presented as the mean \pm standard deviation ( ${ }^{* *} \mathrm{P}<0.01$, vs. NC). SOX18, sex-determining region Y box 18; WT, wild-type; NC, scrambled shRNA transduction; SOX18-Ri-3, SOX18-shRNA-3 virus transduction; PI, propidium iodide; FITC, fluorescein isothiocyanate.

progression between the $\mathrm{S}$-phase and $\mathrm{G}_{2} / \mathrm{M}$-phase, inhibiting apoptosis.

Knockdown of SOX18 inhibits the metastasis of osteosarcoma cells. Metastasis begins with the invasion of tumor cells into the surrounding host tissue. The invasive tumor cells must first alter cell-to-cell adhesion and adhesion to the extracellular matrix (17). The effects of SOX18 on the adherent ability of osteosarcoma cells were evaluated in the present study (Fig. 3A and B). The number of adherent SOX18-Ri-3 cells was $29.5 \%$ of that of the NC cells when U2OS cells were used. Similar results were obtained with the MG63 cells. These data suggested that the adherent ability to fibronectin was significantly inhibited in osteosarcoma cells by SOX18 knockdown.

Whether SOX18 affected the invasive ability of osteosarcoma cells was also investigated using a Transwell assay. As 
A

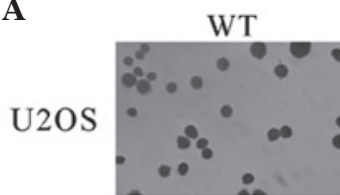

MG63
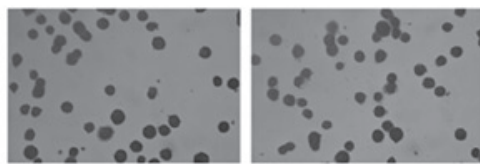

C

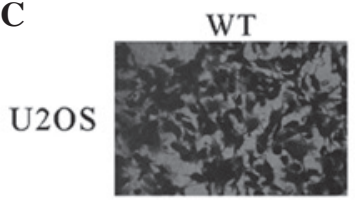

MG63

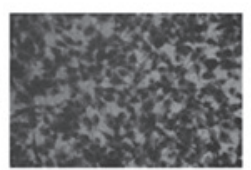

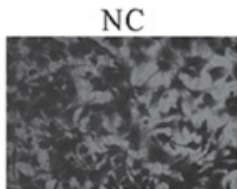

SXO18-Ri-3

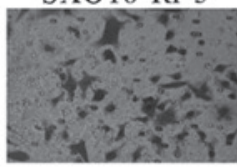

SXO18-Ri-3
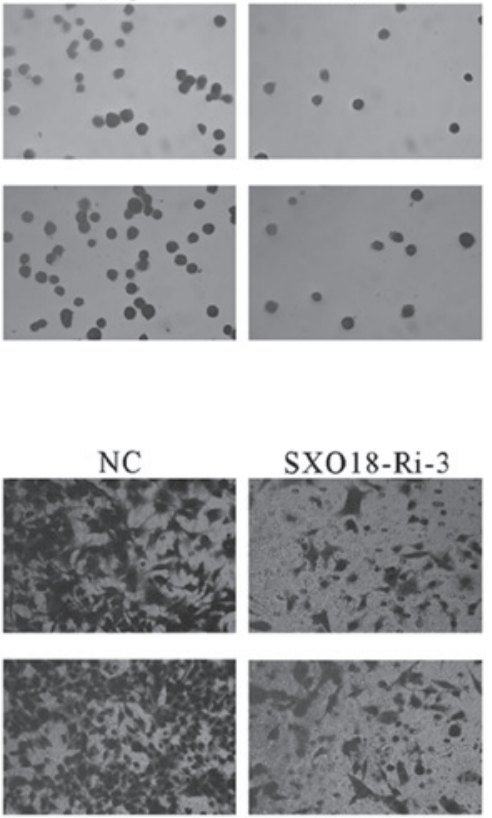

B

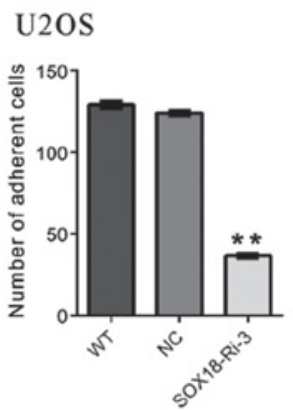

MG63

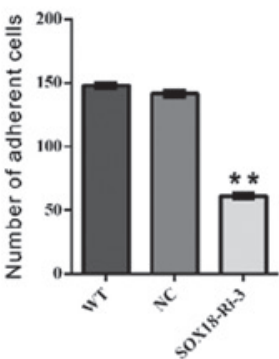

D

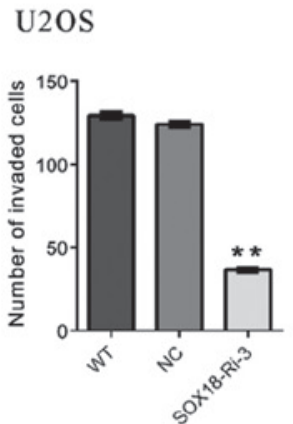

MG63

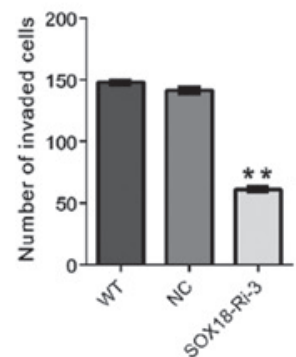

Figure 3. Silencing of SOX18 inhibits the metastasis of osteosarcoma cells and reduces tumor growth in vivo. The U2OS and MG63 cells were transduced with the indicated virus. Adhesion ability was analyzed using a cell adhesion assay. (A) Representative images and (B) quantitative results of the cell adhesion assay. The U2OS and MG63 cells were transduced with the indicated virus and cell invasion was analyzed in Matrigel-coated Transwell chambers. (C) Representative images and (D) quantitative results of the cell adhesion assay. Data are representative of a minimum of three independent experiments and are presented as the mean \pm standard deviation (** $\mathrm{P}<0.01$, vs. NC); magnification, $\mathrm{x} 100$. SOX18, sex-determining region Y-box 18; WT, wild-type; NC, scrambled shRNA virus transduction; SOX18-Ri-3, SOX18-shRNA-3 virus transduction.

A

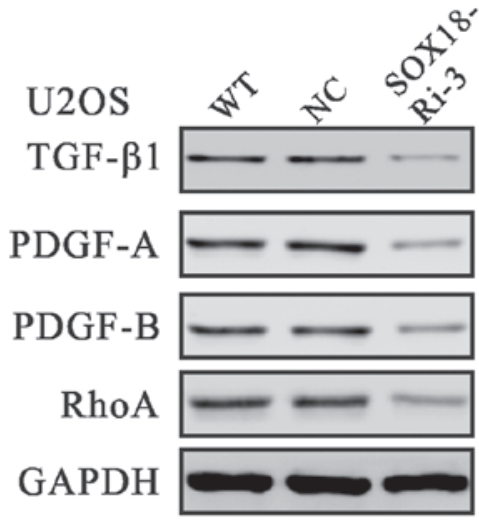

C

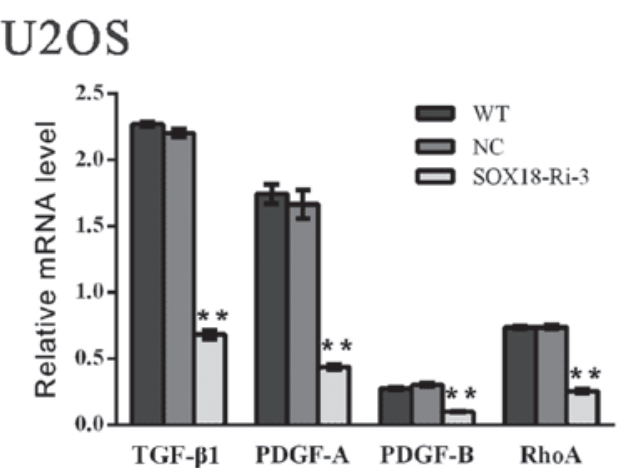

B

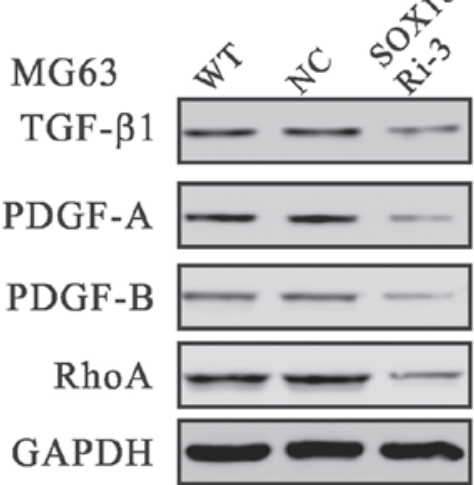

D

\section{MG63}

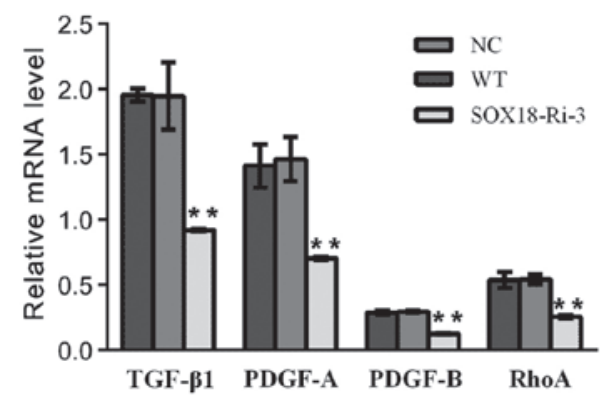

Figure 4. Expression levels of TGF- $\beta 1$, PDGF-A, PDGF-B and RhoA are downregulated by SOX18 RNAi. The protein and mRNA levels of the indicated genes were evaluated using ( $\mathrm{A}$ and $\mathrm{B}$ ) western blotting and $(\mathrm{C}$ and $\mathrm{D})$ reverse transcription-quantitative polymerase chain reaction in the U2OS and MG63 cells. Data are presented as the mean \pm standard deviation $\left({ }^{* *} \mathrm{P}<0.01\right.$, vs. NC). TGF- $\beta 1$, transforming growth factor- $\beta 1$; PDGF-A, platelet-derived growth factor-A; RhoA, Ras homolog family member A; GAPDH, glyceraldehyde 3-phosphate dehydrogenase; SOX18, sex-determining region Y-box 18; WT, wild-type; NC, scrambled shRNA virus transduction; SOX18-Ri-3, SOX18-shRNA-3 virus transduction. 
shown in Fig. 3C and D, transduction of the SOX18-shRNA virus into U2OS or MG63 cells significantly reduced the cell invasion ability, compared with the scramble shRNA (NC). These data suggested that SOX18 promoted osteosarcoma cell invasion.

Expression levels of TGF- $\beta 1, P D G F-A, P D G F-B$ and RhoA are downregulated by SOX18 RNAi. A previous study demonstrated that TGF- $\beta 1$ is a promoter of tumor progression and invasion (18). The classic PDGFs, PDGF-A and PDGF-B, are regarded to be associated with metastasis in various types of human cancer (19-21). It is well known that small GTPase RhoA promotes the invasion of tumor cells (22-24). In order to investigate the molecular mechanisms underlying the role of SOX18 in osteosarcoma cells, the mRNA and protein expression levels of TGF- $\beta 1$, PDGF-A, PDGF-B and RhoA were determined (Fig. 4). The expression levels of all the genes examined were markedly reduced following the downregulation in the expression of SOX18, which suggested that the biological function of SOX18 in osteosarcoma may be associated with these genes.

\section{Discussion}

In the present study, it was found that SOX18 was overexpressed in osteosarcoma. Knockdown of the expression of SOX18 markedly inhibited the transforming ability of osteosarcoma cells. These data indicated the diagnostic and therapeutic value of SOX18 in osteosarcoma.

The involvement of SOX18 in several types of cancer has been an area of investigation, and it has been reported that SOX18 is overexpressed in several types of cancer tissue (10-13), and that SOX18 may promote cellular proliferation $(9,25)$. Garcia-Ramirez et al (25) found that SOX18 is co-localized with the proliferating cell nuclear antigen protein in vascular smooth muscle cells of human coronary atherosclerotic lesions, and that inhibiting the expression of SOX18 results in a reduced proliferation rate in these cells. The expression of dominant-negative SOX18 also reduces the proliferation of human MCF-7 breast cancer cells (9). In the present study, the knockdown of SOX18 in U2OS and MG63 osteosarcoma cells significantly reduced the cell growth rate (Fig. 1). In addition, cell cycle analysis revealed that SOX18 knockdown induced S-phase arrest and apoptosis of osteosarcoma cells (Fig. 2), which may explain the inhibited proliferation of the SOX18-knockdown cells.

Previously, SOX18 was reported to be associated with cell migration and tumor metastasis, and dominant-negative SOX18 was reported to impair the migration of MCF-7 cells (9). Duong et al (26) reported that tumor metastasis is inhibited in SOX18-deficient mice. In line with these observations, the present study found that reduction in the expression of SOX18 in osteosarcoma cells by RNAi significantly reduced their adhesive and invasive capabilities (Fig. 3), indicating that SOX18 may be important in promoting metastasis of osteosarcoma.

The exact pathway that SOX18 may regulate in osteosarcoma remains unclear. TGF- $\beta 1$ has been considered as a promoter of tumor progression and invasion (18). Additionally, PDGFs have been found to induce tumor growth $(27,28)$, and their expression may be useful as a diagnostic and prognostic marker for several types of cancer (29-31). The classic PDGFs,
PDGF-A and PDGF-B, are associated with the metastasis of various types of human cancer (19-21). In the present study, SOX18 RNAi significantly downregulated the expression levels of TGF- $\beta 1$, PDGF-A and PDGF-B, which indicated that SOX18 may execute its functions through regulating the expression of these genes.

It is well known that small GTPase RhoA promotes the invasion of tumor cells (22-24). The expression levels of RhoA may be positively correlated with the progression of carcinoma, suggesting that RhoA may be important in tumorigenesis and tumor progression (32-35). The malignant phenotype in gastric cancer cells (36) and breast cancer cells (37) can be reversed by inhibiting the expression of RhoA. In the present study, it was observed that SOX18 knockdown impaired the expression of RhoA (Fig. 4). Therefore, it was hypothesized that SOX18 may perform its biological function through regulating the expression of RhoA.

In conclusion, the present study demonstrated high expression levels of SOX18 in osteosarcoma, which suggested that SOX18 may be a diagnostic marker for osteosarcoma. The present study suggested for the first time, to the best of our knowledge, that SOX18 is key in the proliferation, apoptosis and metastasis of osteosarcoma cells. In addition, SOX18 may regulate these biological processes through TGF- $\beta 1$, PDGF-A, PDGF-B and RhoA, thus providing potentially useful information for the targeted therapy of osteosarcoma.

\section{References}

1. Ottaviani G and Jaffe N: The epidemiology of osteosarcoma. Cancer Treat Res 152: 3-13, 2009.

2. Provisor AJ, Ettinger LJ, Nachman JB, Krailo MD, Makley JT, Yunis EJ, Huvos AG, Betcher DL, Baum ES, Kisker CT and Miser JS: Treatment of nonmetastatic osteosarcoma of the extremity with preoperative and postoperative chemotherapy: A report from the children's cancer group. J Clin Oncol 15: 76-84, 1997.

3. Bacci G, Ferrari S, Bertoni F, Ruggieri P, Picci P, Longhi A, Casadei R, Fabbri N, Forni C, Versari M and Campanacci M: Long-term outcome for patients with nonmetastatic osteosarcoma of the extremity treated at the istituto ortopedico rizzoli according to the istituto ortopedico rizzoli/osteosarcoma-2 protocol: An updated report. J Clin Oncol 18: 4016-4027, 2000.

4. Rytting M, Pearson P, Raymond AK, Ayala A, Murray J, Yasko AW, Johnson M and Jaffe N: Osteosarcoma in preadolescent patients. Clin Orthop Relat Res 39-50, 2000.

5. Ferguson WS and Goorin AM: Current treatment of osteosarcoma. Cancer Invest 19: 292-315, 2001.

6. Hosking BM, Muscat GE, Koopman PA, Dowhan DH and Dunn TL: Trans-activation and DNA-binding properties of the transcription factor, Sox-18. Nucleic Acids Res 23: 2626-2628, 1995.

7. Hosking BM, Wyeth JR, Pennisi DJ, Wang SC, Koopman P and Muscat GE: Cloning and functional analysis of the Sry-related HMG box gene, Sox18. Gene 262: 239-247, 2001.

8. Darby IA, Bisucci T, Raghoenath S, Olsson J, Muscat GE and Koopman P: Sox 18 is transiently expressed during angiogenesis in granulation tissue of skin wounds with an identical expression pattern to Flk-1 mRNA. Lab Invest 81: 937-943, 2001.

9. Young N, Hahn CN, Poh A, Dong C, Wilhelm D, Olsson J, Muscat GE, Parsons P, Gamble JR and Koopman P: Effect of disrupted SOX18 transcription factor function on tumor growth, vascularization and endothelial development. J Natl Cancer Inst 98: 1060-1067, 2006.

10. Eom BW, Jo MJ, Kook MC, Ryu KW, Choi IJ, Nam BH, Kim YW and Lee JH: The lymphangiogenic factor SOX 18: A key indicator to stage gastric tumor progression. Int J Cancer 131: 41-48, 2012.

11. Jethon A, Pula B, Olbromski M, Werynska B, Muszczynska-Bernhard B, Witkiewicz W, Dziegiel P and Podhorska-Okolow M: Prognostic significance of SOX18 expression in non-small cell lung cancer. Int J Oncol 46: 123-132, 2015. 
12. Pula B, Kobierzycki C, Solinski D, Olbromski M Nowak-Markwitz E, Spaczynski M, Kedzia W, Zabel M and Dziegiel P: SOX18 expression predicts response to platinum-based chemotherapy in ovarian cancer. Anticancer Res 34: 4029-4037, 2014.

13. Pula B, Olbromski M, Wojnar A, Gomulkiewicz A, Witkiewicz W, Ugorski M, Dziegiel P and Podhorska-Okolow M: Impact of SOX18 expression in cancer cells and vessels on the outcome of invasive ductal breast carcinoma. Cell Oncol (Dordr) 36: 469-483, 2013

14. Livak KJ and Schmittgen TD: Analysis of relative gene expression data using real-time quantitative PCR and the 2(-Delta Delta C(T)) Method. Methods 25: 402-408, 2001.

15. Henry MK, Lynch JT, Eapen AK and Quelle FW: DNA damage-induced cell-cycle arrest of hematopoietic cells is overridden by activation of the PI-3 kinase/Akt signaling pathway. Blood 98: 834-841, 2001.

16. Silletti S, Paku S and Raz A: Autocrine motility factor and the extracellular matrix. I. Coordinate regulation of melanoma cell adhesion, spreading and migration involves focal contact reorganization. Int J Cancer 76: 120-128, 1998.

17. Mareel M, Oliveira MJ and Madani I: Cancer invasion and metastasis: Interacting ecosystems. Virchows Arch 454: 599-622, 2009.

18. Wakefield LM and Roberts AB: TGF-beta signaling: Positive and negative effects on tumorigenesis. Curr Opin Genet Dev 12: 22-29, 2002

19. Kodama M,Kitadai Y, Sumida T, Ohnishi M, Ohara E, Tanaka M, Shinagawa K, Tanaka S, Yasui W and Chayama K: Expression of platelet-derived growth factor (PDGF)-B and PDGF-receptor $\beta$ is associated with lymphatic metastasis in human gastric carcinoma. Cancer Sci 101: 1984-1989, 2010.

20. Donnem T, Al-Saad S, Al-Shibli K, Busund LT and Bremnes RM: Co-expression of PDGF-B and VEGFR-3 strongly correlates with lymph node metastasis and poor survival in non-small-cell lung cancer. Ann Oncol 21: 223-231, 2010.

21. Jechlinger M, Sommer A, Moriggl R, Seither P, Kraut N, Capodiecci P, Donovan M, Cordon-Cardo C, Beug H and Grünert S: Autocrine PDGFR signaling promotes mammary cancer metastasis. J Clin Invest 116: 1561-1570, 2006.

22. Struckhoff AP, Rana MK and Worthylake RA: RhoA can lead the way in tumor cell invasion and metastasis. Front Biosci (Landmark Ed) 16: 1915-1926, 2011.

23. Yan G, Zou R, Chen Z, Fan B, Wang Z, Wang Y, Yin X, Zhang D, Tong L, Yang $\mathrm{F}$, et al: Silencing RhoA inhibits migration and invasion through $\mathrm{Wnt} / \beta$-catenin pathway and growth through cell cycle regulation in human tongue cancer. Acta Biochim Biophys Sin (Shanghai) 46: 682-690, 2014.

24. Yoshioka K, Nakamori S and Itoh K: Overexpression of small GTP-binding protein RhoA promotes invasion of tumor cells. Cancer Res 59: 2004-2010, 1999.
25. Garcia-Ramírez M, Martinez-González J, Juan-Babot JO, Rodríguez C and Badimon L: Transcription factor SOX18 is expressed in human coronary atherosclerotic lesions and regulates DNA synthesis and vascular cell growth. Arterioscler Thromb Vasc Biol 25: 2398-2403, 2005.

26. Duong T, Proulx ST, Luciani P, Leroux JC, Detmar M, Koopman P and Francois M: Genetic ablation of SOX18 function suppresses tumor lymphangiogenesis and metastasis of melanoma in mice. Cancer Res 72: 3105-3114, 2012.

27. Ostman A: PDGF receptors-mediators of autocrine tumor growth and regulators of tumor vasculature and stroma. Cytokine Growth Factor Rev 15: 275-286, 2004.

28. Uehara H, Kim SJ, Karashima T, Shepherd DL, Fan D, Tsan R, Killion JJ, Logothetis C, Mathew P and Fidler IJ: Effects of blocking platelet-derived growth factor-receptor signaling in a mouse model of experimental prostate cancer bone metastases. J Natl Cancer Inst 95: 458-470, 2003.

29. Sulzbacher I, Birner P, Trieb K, Träxler M, Lang S and Chott A: Expression of platelet-derived growth factor-AA is associated with tumor progression in osteosarcoma. Mod Pathol 16: 66-71, 2003.

30. Peterson JE, Zurakowski D, Italiano JE Jr, Michel LV, Connors S, Oenick M, D'Amato RJ, Klement GL and Folkman J: VEGF, PF4 and PDGF are elevated in platelets of colorectal cancer patients. Angiogenesis 15: 265-273, 2012.

31. Ariad S, Seymour L and Bezwoda WR: Platelet-derived growth factor (PDGF) in plasma of breast cancer patients: Correlation with stage and rate of progression. Breast cancer Res Treat 20: 11-17, 1991

32. Abraham MT, Kuriakose MA, Sacks PG, Yee H, Chiriboga L, Bearer EL and Delacure MD: Motility-related proteins as markers for head and neck squamous cell cancer. Laryngoscope 111: 1285-1289, 2001.

33. Horiuchi A, Imai T, Wang C, Ohira S, Feng Y, Nikaido T and Konishi I: Up-regulation of small GTPases, RhoA and RhoC, is associated with tumor progression in ovarian carcinoma. Lab Invest 83: 861-870, 2003.

34. Kamai T, Arai K, Tsujii T,Honda Mand Yoshida K: Overexpression of RhoA mRNA is associated with advanced stage in testicular germ cell tumour. BJU International 87: 227-231, 2001.

35. Kamai T, Tsujii T, Arai K, Takagi K, Asami H, Ito $\mathrm{Y}$ and Oshima H: Significant association of Rho/ROCK pathway with invasion and metastasis of bladder cancer. Clin Cancer Res 9: 2632-2641, 2003

36. Liu N, Bi F, Pan Y, Sun L, Xue Y, Shi Y, Yao X, Zheng Y and Fan D: Reversal of the malignant phenotype of gastric cancer cells by inhibition of RhoA expression and activity. Clin Cancer Res 10: 6239-6247, 2004.

37. Pillé JY, Denoyelle C, Varet J, Bertrand JR, Soria J, Opolon P, Lu H, Pritchard LL, Vannier JP, Malvy C, et al: Anti-RhoA and anti-RhoC siRNAs inhibit the proliferation and invasiveness of MDA-MB-231 breast cancer cells in vitro and in vivo. Mol Ther 11: 267-274, 2005. 